\title{
Telemetry: connectivity and productivity in real time - project implementation guide
}

\author{
R Marín Sandvik, Chile
}

\begin{abstract}
Over the past decade, the mining industry has faced increasingly complex global changes and operating conditions. These situations have led to changes in strategy and altered courses of action, and necessitated the adoption of new business and operational models. Globalization, the opening of new markets, volatility in commodity prices, ever deeper mines, greater travel distances and cost increases, coupled with more extreme operating conditions and ever-lower average ore grades, have generated a challenging environment. In order to remain competitive and viable it is imperative for the mining industry to act quickly and adapt to new demands. According to McKinsey (2015) research, worldwide mining operations are as much as 28 percent less productive today than a decade ago. It is a fact that we are now experiencing the fourth industrial revolution (Industry 4.0) which has a general impact on all industries and levels within a company. It is ushering in new stage of connectivity between the physical and digital worlds of intelligent devices (IOT), large volume data generation and management (Big DataCloud Computing), autonomous systems and equipment operation, and Al (artificial intelligence/machine learning) for dynamic analysis. Digital transformation is no longer the privilege of a few, but a necessity for all in order to be competitive, and mining is not an exception. One area of recent exponential growth is telemetry, which allows mines to have equipment connectivity in real-time. With telemetry, technology critical operational data can be recorded and then used for accurate process analysis, optimal fleet management and mining asset maintenance. Telemetry has great potential to innovate mining, but it is up to the industry to take the initiative and embrace it for more efficient processes, and to make more effective decisions and increase productivity. This document aims to share guidelines and practical experiences for implementing telemetry as a tool. Further, it will demonstrate some of its benefits for fleet equipment management in underground mining operations, and show how it can extend interoperability concepts in comprehensive asset management under the same management platform.
\end{abstract}

\section{Introduction}

It is interesting to be protagonists in events that mark the beginning of trends or great changes in human history. Advances in science, economic recessions and fluctuation, global pandemics, political change, among so many others, are shaping the future for generations to come. The fourth industrial revolution and its corresponding technological innovation is fast approaching our homes, workplaces and industries, while exponentially opening up new opportunities for the global community and, in particular, for mining companies.

The unprecedented COVID-19, which began at the end of 2019, has put humanity to the test and made it clear that technology is a necessary and essential resource for dealing with complex scenarios. Agility, preparation, adaptation and assimilation of technology are necessary to deal with these situations of disruption and fear of the unknown. 
According to a World Economic Forum (2017), by 2025 the mining industry will see the greatest technological development and impact in the areas of automation and robotics, digitally enabled workforces, platform integrations, ecosystem and business analytics, and artificial intelligence for realtime decisions. The internet of things (IOT) and real-time analytics will play an important role in this revolution across industries and business processes. The study goes on to say that this will be a paradigm change for all industries on the planet and that new technologies will be disruptive, such as telework, or transformative, such as telemetry.

Telemetry is undoubtedly one of the technologies that has had a cross-cutting impact on various applications and industries. However, in mining it is just beginning to establish itself as a mechanism for the control and management of operational and maintenance processes. Sandvik Mining and Rock Technologies (SMRT) has developed a comprehensive portfolio of solutions focused on digitalization technologies over the last few decades. Among these are the teleoperation and autonomous operation of equipment and information management software packages. Current telemetry solutions allow an active fleet of more than $\mathbf{2 0 0 0}$ units to be connected to the cloud with Sandvik equipment, and enable effective management in real-time. The scope and integration of multi-brand equipment selection has been amplified by their acquisition of Newtrax Technologies Inc. This substantially favors integration and interoperability of the entire fleet of equipment on a single platform, without brand or type distinction.

This paper seeks to give a practical approach to the process of implementing telemetry projects. It uses as its framework the Global Mining Guidelines Group (2019) "Guidelines for Implementing Short Interval Control in Underground Mining Operations" publication, which considers three fundamental axes: technology, process and people. Often the actual mining project situation and the company's desired situation are not the same. The first step is a fundamental analysis with the customer's participation in order to visualize and understand all considerations. The result of this step should be a verification that the company is prepared to advance to a digital transformation (or not) and/or a plan to resolve basic preliminary aspects in advance of the implementation.

\section{$2 \quad$ What is telemetry?}

Telemetry is one of the most quickly advancing applications in analytics. Etymologically, the word telemetry is of Greek origin, "tele" meaning "distance", and "metria", expressing "measure". Telemetry is the remote measurement of any physical input and the subsequent sending of that information through a communication channel for later registration, control and monitoring. Any telemetry system has at least three parts: the physical input to measure, a communication channel and a control unit. As defined by the GMG, telemetry is an automated technological process for communicating measurements and other data between remote locations and receiving equipment.

A telemetry system uses a transducer or sensor as an input device, a wireless or fixed transmission medium, signal processing devices, and data storage or display devices. The transducer converts a physical input such as temperature, pressure or time into a corresponding electrical signal, which is transmitted from a remote location for measurement and recording purposes.

For underground mining equipment the physical inputs to be measured vary depending on the equipment and the registration objectives and each of these variables are unique. Variables that might be measured for a Jumbo, LHD or a low-profile truck, for example, could include temperature, motor RPM, motor horometer readings, and fuel consumption. The system also records information such as percussion hours, percussion pressure in the case of Jumbos, or oil transmission pressure for LHDs and trucks. The telemetry solution for underground fleet management developed by Sandvik is called My Sandvik and its architecture is shown in Figure 1. 


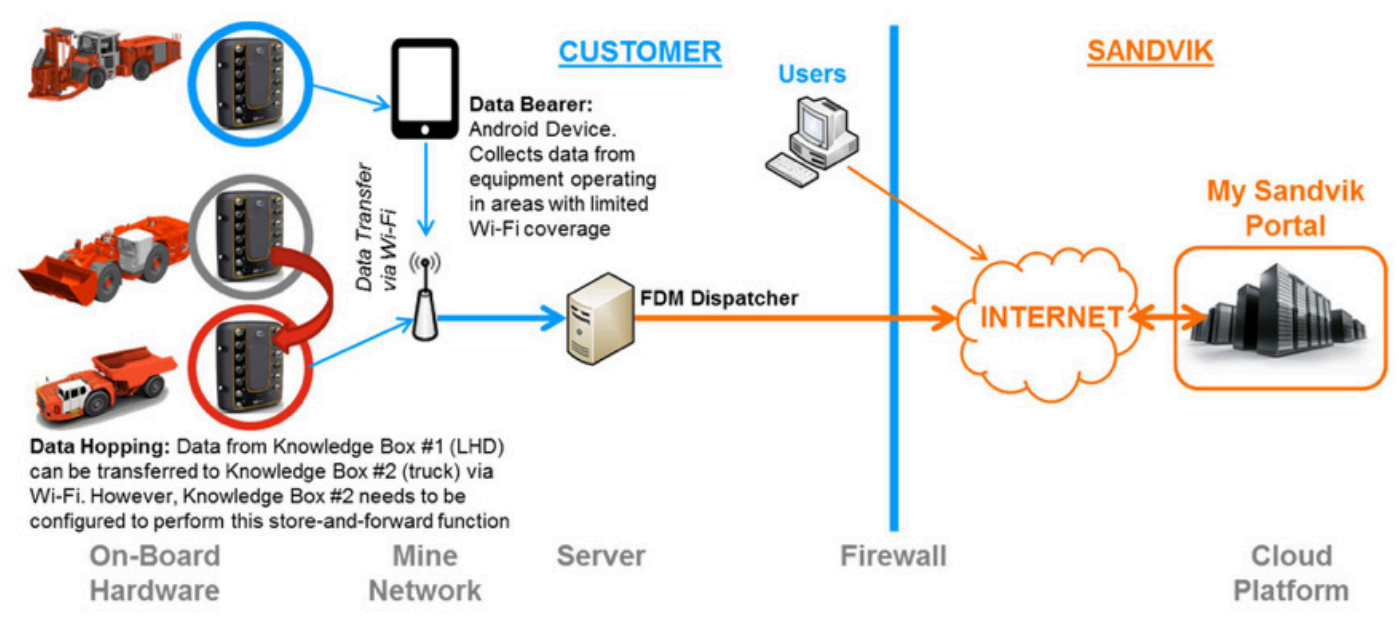

Figure 1 Cloud-based (My Sandvik) monitoring system

\section{$2.1 \quad$ On-board hardware}

Data is recorded through an on-board device called the Knowledge Box, which collects information with sensors from the equipment and translates it into a code for database registration. The Knowledge Box is a rugged industrial PC, with built-in Wi-Fi capability. It provides near real-time communication for collecting essential equipment data for production, condition and equipment utilization monitoring. The Knowledge Box generates a compressed data file every 5 minutes (by default), which contains the signal data collected from the equipment, and which varies by functionality. For example, fast varying signals such as engine RPM are sampled every 5 seconds. Additionally, it is designed to 'store-and-forward' the data collected, and automatically uploads the data file to the server when the equipment comes into Wi-Fi coverage.

\section{$2.2 \quad$ Mine network}

The current trend for communication channels is to have wireless coverage in the areas of operation, however factors such as distance, sectors with little service availability and/or difficulty in access must be considered. Benefits of Wi-Fi include the flexibility of not having cables, its low cost, and the possibility of viewing the information later on a tablet, laptop, or other mobile device.

At present, the Sandvik Knowledge box supports IEEE $802.11 \mathrm{~b} / \mathrm{g}$ protocols (i.e. most commonly used standard wireless devices for home, office and industrial applications). The Wi-Fi access points used for monitoring need to support these protocols.

To provide up-to-date data from the equipment, it is recommended that the equipment should come within the Wi-Fi coverage area once per cycle (in the case of loaders or trucks) or, at minimum, once per shift. To achieve communication that is closer to real-time, Wi-Fi access points can be installed throughout the production area, although this is not usually necessary.

\subsection{Data transfer to cloud}

As a cloud-based solution, My Sandvik requires the equipment data to be transferred to the internet cloud platform. Therefore, data security is critical and encryption is used whenever data is transferred to the cloud platform.

For improved user experience, and faster data transfer to their cloud platform, Sandvik recommends installing the 'FDM Dispatcher' application on a server. The FDM Dispatcher application acts much like a buffer. With a single security authentication, the FDM Dispatcher allows simultaneous transfer of multiple data files to the cloud platform. 


\section{$2.4 \quad$ Cloud platform}

The control unit is responsible for receiving the transmitted information, processing it according to builtin business logic and then sharing it on dashboards and monitoring tools in real-time. Through the My Sandvik portal, users can access the cloud-based monitoring system from any device with Wi-Fi access.

Monitoring and reporting take in to account the following factors:

- Equipment production, productivity and performance reports

- Utilization reports, equipment operating status with a defined time horizon

- Alert reports, warnings and operator events, limits and warnings regarding the defined equipment parameters. Additionally, verify and improve practices in equipment operation

- Signal equipment reports, descriptive information on machine health broken down by equipment type and major components. This is especially relevant for maintenance planning and diagnostics

Telemetry is a clear example of a connectivity application between physical and digital worlds with a focus on improving productivity, efficiency, security and business profitability. In the past, and in some cases even now, engine hours for LHD production equipment or the hours of equipment used must be manually recorded, even though technology is now available. The change to telemetry systems would optimize these process chains and more effectively focus resources and decision-making by providing relevant analysis based on reliable, real-time information.

\section{$3 \quad$ Implementing a telemetry system}

The monitoring of equipment through telemetry in mining, with its real-time analytics, has quickly become an important support tool for informed decision making where data is a valuable asset. Today, many operations continue to operate in the "old school" way, by manually recording equipment data and operations, with no measurements that allow control based on quantifiable data. Manual recording of operational data, excessive paperwork, and lack of periodic controls on variables and critical processes are just a few of factors that have increased maintenance costs. Moreover, they can tribute to component life or component failure and affect equipment availability issues. In enterprises where Sandvik technology has been deployed there has been a mitigation of these affects.

Data capture and logging, until recently, was not efficiently manageable in large volumes. The technology has continuously advanced and now facilitates efficient information management and business intelligence platform use without human interaction and/or manual records. Resources can now be focused on systematic analysis and qualitative expert judgment of information already captured and processed, as well as on advanced predictive algorithms based on historical behavior patterns. This process eliminates manual data capture, potential data processing errors, and information corruptibility, while at the same time addressing possible loss issues and security.

While this technology has a general impact within the organization, the areas of maintenance and operations are the main beneficiaries of these single sources of input for equipment operation. This becomes apparent in a case where management utilization reports are generated manually for an active fleet of equipment. There may be the assumption that the source of information is the same, when in fact the report results have no relationship. This can generate controversy; waste time and credibility can suffer. The effects are multiplied as this misinformation reaches higher levels of the mine management, which has a more complete perspective on operations.

Sandvik's vision and experience are in complete alignment with the Global Mining Guidelines Group (2019), based on Short Interval Control (SIC). This lean process approach delivers a series of guidelines for gradual implementation, with efficient and effective resource allocation subject to a continuous 
improvement process (PDCA, Plan-Do-Check-Act). One of the central components is the periodic review (three or four times per shift) of the actual versus planned results in order to check for deviations and adjust the target. This is how waste is minimized and productivity increased.

In addition, it is important to identify the maturity level of technology within the organization, so that a base level from which to continue working on information, integration, automation, and analytics for decision-making, among others, are clearly established. Telemetry systems often interact with other platforms, whether they are location tracking modules of equipment, people or other systems that require some deliverable input which they must generate. In many cases the pre-implementation project interviews indicate that the company is not yet prepared to take the steps to digital transformation, but first must solve a number of problems to meet the requirements. In these cases, it is more convenient to take a step back than to move forward. In other words, the company should allocate resources to work on the preliminary requirements identified and not divert them toward a digital solution for which is not ready.

The following implementation model considers three fundamental stages of an SIC technology project.

\subsection{Current stage ("As-Is")}

To start, it is essential to diagnose the current operational scenario of the mine and its primary characteristics. It is necessary to be clear about the projection of the operation and mine life, safety conditions, day-today operating conditions, strategic projections and targets, and resources and assets available (processes, equipment, people, software, hardware, management platforms, spare parts, supplies, tools, etc.)

According to the SIC (GMG), the objective is to adapt to changing conditions while remaining as close as possible to the plan. This entails a continuous drive toward improvement based on data and recorded events in order to ensure that the operational plans are based on the most complete and precise scenario.

It is essential to prioritize the equipment monitoring data that is needed to meet the goals and deliverables that have been committed to. Many companies perform a manual data capture of the operational conditions and of equipment data on log sheets, which are then transferred to an Excel-like information sheet. No doubt the probability of error, corruptibility and loss of information is high. Added to this is the fact that on many occasions there is no defined periodicity nor is there a formally established equipment monitoring process. It is necessary to have clarity for all the information mapping, from its origin, through its flow, and to its final destination. It is critical to identify, or to have:

- The origin of the data and who is responsible for recording it.

- The data entry format.

- How often data is collected.

- How data is captured and delivered.

- The ability to understand, analyze and leverage the information generated.

- IT infrastructure, such as Wi-Fi UG network coverage.

- Personnel that are knowledgeable about the process and new technologies, such as telemetry.

- A staff profile for those to be involved in the process.

- The opinions and positions of operators, shift managers, maintenance staff and senior managers on including technologies in the operation.

- The types of existing controls (e.g. standards, protocols) or governance (e.g. meetings, event triggers) that influence how, when, and in what format data is generated.

- The output data format and destination (for example, the interaction and relationship with other areas and/or processes). 
- The existence of goals, processes, managers, reports and process management by KPIs. For example, check for defined reports and goals and of availability and utilization of equipment by fleet type, loading and hauling fleet fuel consumption, and for meters drilled by shift in the case of Jumbos.

- Improvement action plans in response to plan deviations that are shown by the periodic reports (during the shift, at the close of the shift, by day, by month).

- Benchmarking from other mine-operations and identification of the maturity level of the operation.

\subsection{Gap analysis and target identification}

At the next stage, gaps between the current situation ("As-Is") and a desired future ("To-Be") must be identified by performing a comparison study of similar industry operations. In particular, the level of existing maturity versus the aim are critical. For the maturity level of a SIC implementation, the GMG sets useful guidelines with 6 levels. Maturity levels are described on Figure 2.

Most telemetry project implementation cases start at level 1 or level 2 . The idea is to design a gradual scenario that advances over time, continually adding value to the process and ultimately achieving the goal.

Level 1: Basic. Manual data capture and end-of-shift reporting.

Level 2: Foundation. Includes some digital data capture, though not in real-time; no connectivity between systems.

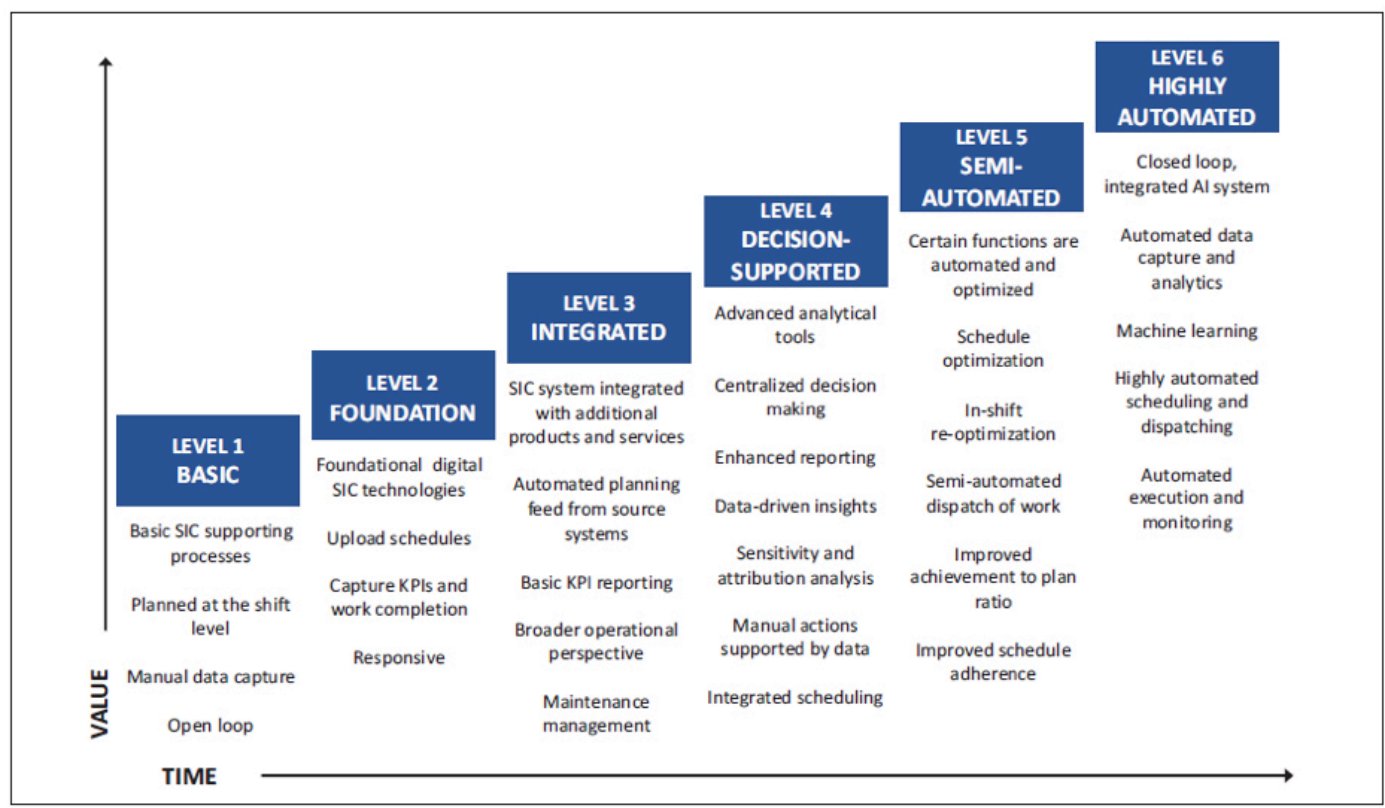

Figure 2 Short interval control (SIC) system maturity levels

\section{Pilot and simulations as an alternative to starting point}

Prior to the formal implementation of a telemetry system an intermediate step is possible in order to gain experience and maturity in the fleet management technology. Several operations have chosen the option of simulations and pilot projects. Both options offer a fast track evaluation of the technology and greater familiarity with the scope of the main requirements and further deliverables that could be addressed. Pilot tests are set up with a defined operating time, selection of a representative fleet to monitor and target definition. Once the pilot test is finished, a result review workshop is held for feedback, and the results are extrapolated to another situation depending on the user's acceptance. While pilot projects are useful for the purposes described, they are recommended for evaluation periods of not less than 6 months, due to the length of start-up and adaptation periods. 
A pilot project is currently underway on a massive underground operation in Chile, in which a series of data diagnostics were carried out in the first stage. A work plan is being generated to expand the possibilities of more intensive use of the tool in order to bring the organization to a higher maturity level. Customer feedback has been positive in the sense that the data being generated can answer several questions that the operation currently has. The main focus was to show the potential of the collected information and how to interpret and use it in a proactive way for operational and maintenance decisions.

A fleet of 17 LHDs was selected for an information log review for a period of around 1 week (from January 24 to January 30) to verify operational indicators and health information from each piece of equipment. The following drivers were analyzed:

- Utilization. Operating status of LHDs over a week of analysis.

- Alarms/Events. For engine delayed shutdown interruption and safety violations as a result of inappropriate equipment operation that could cause component damage.

- Signals. 80/20 descriptive information analysis of transmission oil temperature.

Relevant operational and equipment condition data was collected by the on-board knowledge box, and then transferred and stored in a cloud database to be analysed. Some dashboard examples delivered by the telemetry application and shared with the customer for further discussions are summarized in the following section.

\subsubsection{Safety violations}

Main concept: Inappropriate operation of the LHD during tramming, including the following operator events highlighted in figures 3 and 4 :

- Braking violation: brake and throttle pedals are pressed, and the travel speed is greater than 5 $\mathrm{km} / \mathrm{h}$ for 5 seconds.

- Freewheel in neutral: the travel speed is greater than $5 \mathrm{~km} / \mathrm{h}$ and the neutral gear is selected for 3 seconds.

- Parking brake violation: the travel speed is greater than $5 \mathrm{~km} / \mathrm{h}$ when the parking brake button is pressed.

- Speeding: travel speed is greater than $20 \mathrm{~km} / \mathrm{h}$ for 5 seconds.

Probable Impact: Excessive events can cause premature damage to brake discs

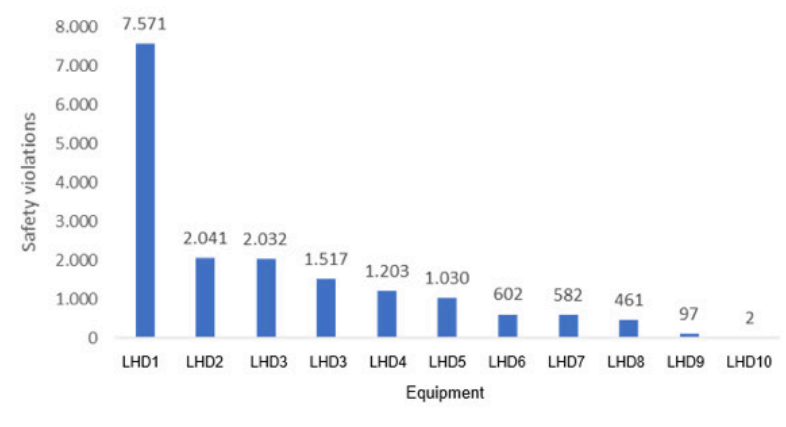

Figure 3 Events by equipment

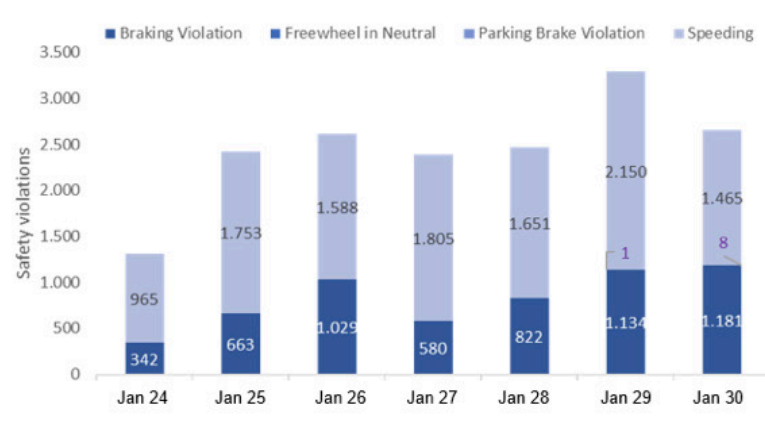

Figure 4 Events by day

Potential Improvement Actions: According to figures 3 and 4, several events were register during the analyzed period and a major concentration is in LHD1. Therefore, a special operator training campaign could address correct equipment operation and reduce the number of events. The study also offered insight in to the frequency with which operators exceed the speed limit. This information is very important in terms creating culture of safety at the mine site, for eliminating hazards and accidents. 


\subsubsection{Alarms for engine delayed shutdown interruption}

Main concept: Inappropriate shutdown of the engine affecting the turbo, including the following operator events highlighted in figures 4 and 5 :

Type of alarms considered:

- Chair 68 - Delayed engine shutdown aborted. Door open!

- Chair 69 - Delayed engine shutdown aborted. E-stop active!

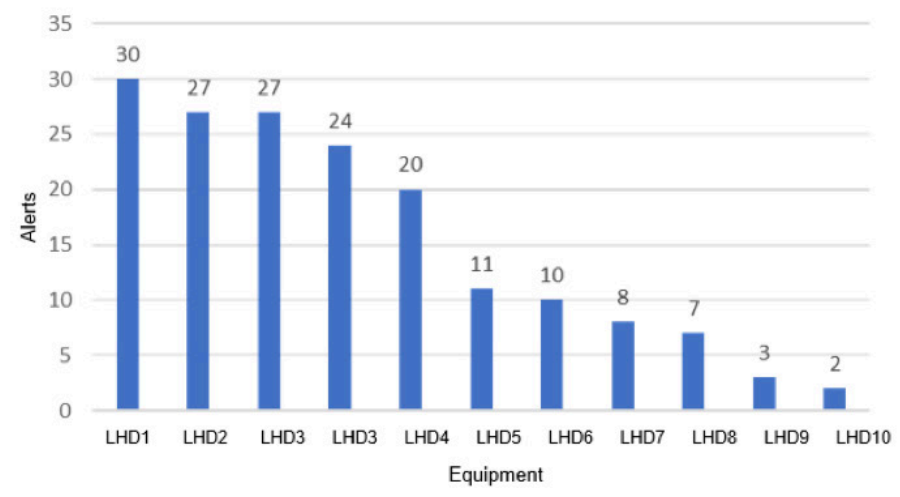

Figure $5 \quad$ Alarms by Equipment

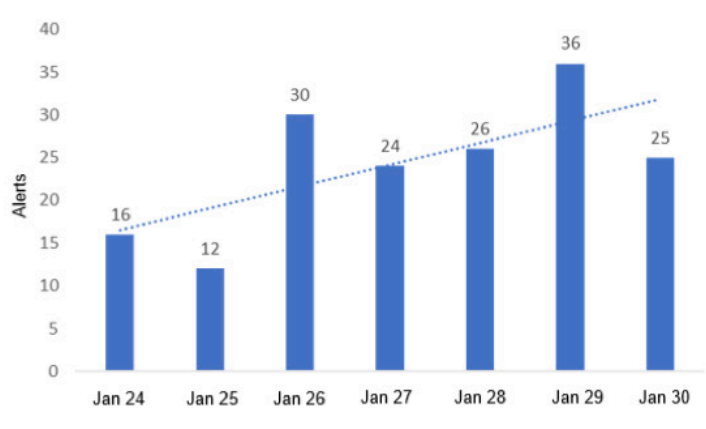

Figure 6 Alarms by day

Probable impact: affects the state and cycle life of the turbo

Potential Improvement Actions: According to figures 4 and 5, several registered events showed an increase over the week as well as more cases in the first 5 LHDs. Therefore, special operator training campaigns could be address correct equipment operation and reduce the number of events.

\subsubsection{Transmission oil temperature diagnostic}

Pareto analysis:

- Middle 142- 143: transmission oil temperature, high warning $\rightarrow 37 \%$.

- Middle 51: steering valve signal wire broken (no feedback) $\rightarrow 9 \%$.

- Chair 28: E-stop activated $\rightarrow 9 \%$.

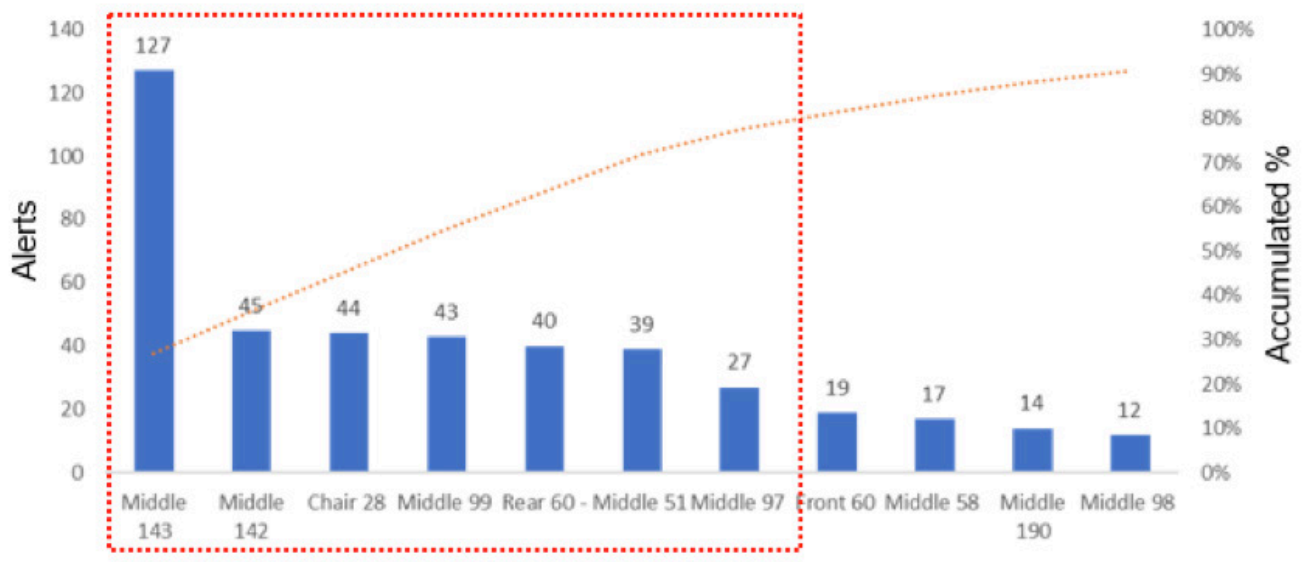

Figure 7 Pareto analysis for alarms

Below are recommendations for further actions. 
Middle 142-143: The current value of the temperature sensor is very high:

- Check filter status and oil analysis

- Check for leaks and transmission oil level

- Check main transmission pressure (240 to 280 PSI)

- Perform working pressure tests on gear and speed drums (without pressure drops of minus 5 PSI)

Alarms: Figure 8 shows transmission oil temperatures registered during the week of analysis based on the pre-established alarm and warning limits which triggered the signals.

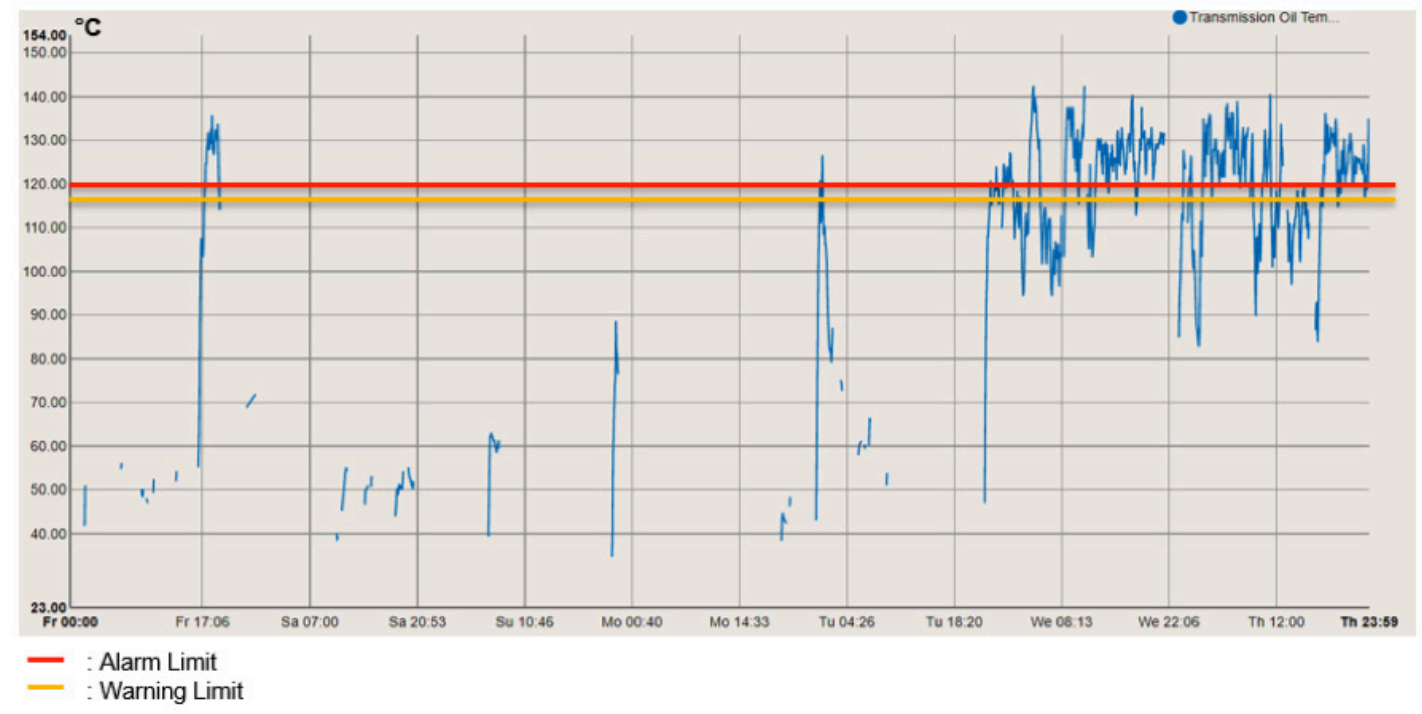

Figure 8 Equipment signals: transmission oil temperature

\subsection{Future state ("To-Be")}

Once the current situation has been identified, it is necessary for all the stakeholders to agree on the meaning of a successful implementation with SIC. Because the SIC model is based on continuous improvement at short-range intervals, revisions needed because of deviations (identified with real-time information) are made at shift closing or during the shift, closing the issue. Ideal future situations could include:

- Mapping of the desired structure and flow of information.

- Process automation levels and definition of future KPIs for process control.

- Comparison to existing KPIs.

- Definition/redefining of roles, processes, responsibilities (operators, shift managers, reliability analysts, mine manager, operations manager, maintenance manager, etc.).

- Integration with other platforms (IT systems and upgrade plans).

A detailed schema with suggested actions to be addressed is shown below in Figure 9. The 3 relevant drivers to take into consideration for a technology project like telemetry assessment are: people, process and technology. Nowadays the success of any digital project implementation should take into account change management and how these three variables work and depend on each other. Is not only about the availability of technology, and it is not just a "plug and play" process. It is more about human behavior and how people integrate technology into their operations. 


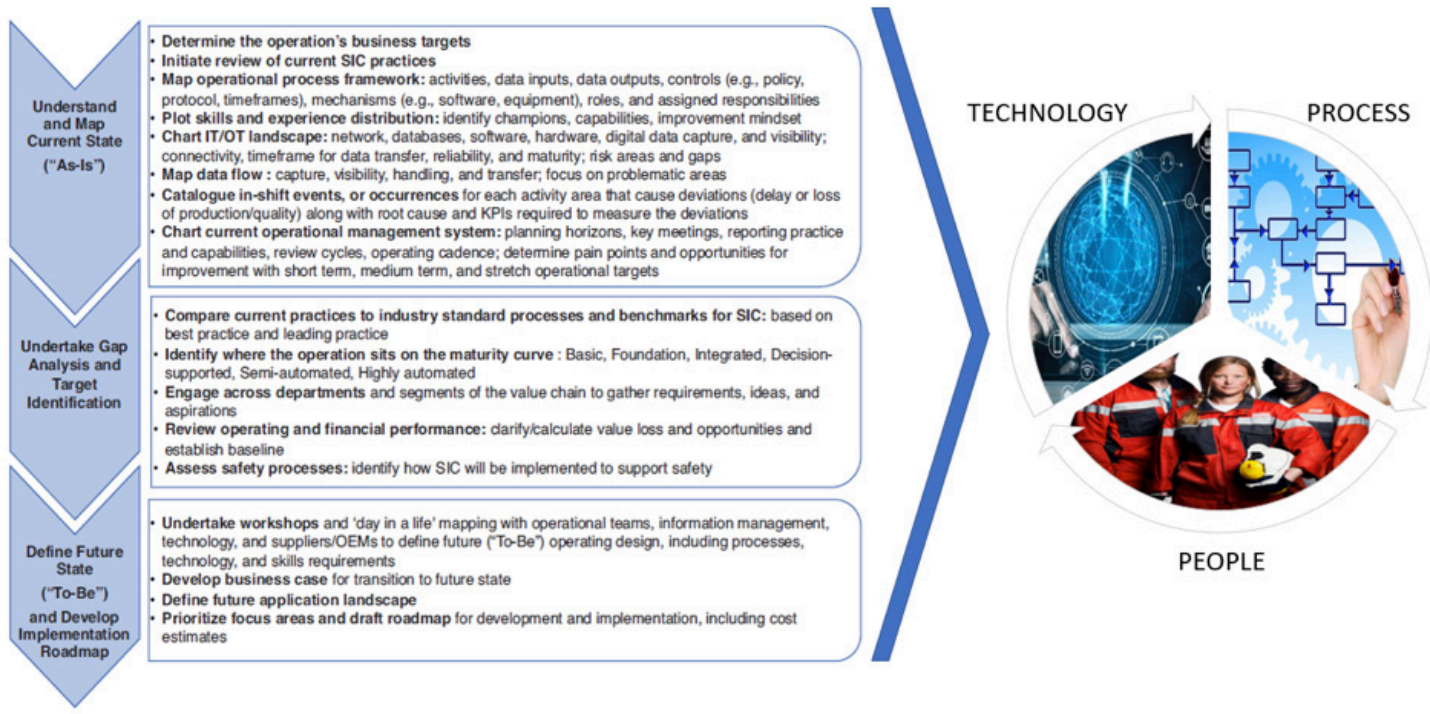

Figure 9 Conceptual process to develop a Short Interval Control (SIC) framework - GMG with adaptation

\subsection{Activities impacted by SIC}

Some activities and processes that benefit as a result of the SIC implementation are described below:

- Short-term plan. Planning becomes more efficient, evaluating input productivity information and equipment availability in real time.

- Planning and execution. Potential integration of complementary processes and platforms. Planning the task detail assignments for certain resources (process, operator, equipment, fleet) to comply with the plan. Execution is fed back from an equipment monitoring platform with realtime production, which is recorded and shared via a control room. The impact of this information is assessed, and short-term plan changes verified.

- Maintenance plan. Facilitates the preparation of future maintenance plans with descriptive health and operational equipment information registered in the monitoring system. The goal is to have maintenance guidelines with a look toward preventive maintenance that prioritizes change of critical components according to real-time operating information. For example, the life extension of equipment and major components and the optimization of spare parts management in terms of timely sourcing and efficient storage could be achieved.

- Measurement. Data recording is carried out automatically from sensors and on-board equipment, and tablets feed information directly to a database without intervention from third parties. This ensures confidence in the quality of data in contrast to a manual operation. Similarly, out-ofservice equipment health alert information could result in a change in the production plan or a recognition of new safety issues,

- Analysis Approach. As opposed to administrative or data capture tasks which do not add value to the process. Real-time information (depending on the refresh rate) with different aggregation levels over time (shift, day, month, year) and historical data records. Global vision of fleet equipment, with the possibility of detecting patterns and isolating particular behaviors agilely. Performance data is available so operators can track their progress with respect to the plan, frontline managers can track plan delivery (the short-term plan) and other stakeholders can ensure that short-term plan execution is transparent.

- Decision support. This process undoubtedly benefits front line supervisors, shift managers and operators, allowing them to make decisions based on quality and real-time data. Improvisation is set aside, and corrective actions can be made in the moment. An expert approach to adjustment for deviations in a short-term production plan or a potential early diagnosis of equipment that is out of service can be taken. 


\subsection{Implementation with cross-cutting approach: technology, people and processes}

This implementation process has a cross-cutting approach to three interrelated central axes, which should be identified and considered in technological implementation project. These are: technology, people and processes. Based on Sandvik's experience in the implementation of such technologies, critical success relates to focusing attention on and tracking these three variables not only during the project implementation phase, but once it has been delivered and also later as its use matures. The following are several key points identified through user interviews at different phases of telemetry project deployment:

- Technology. Today's telemetry technology is proven to work with the large amount of data available for direct use and transformation. Advancement continues in platform integration, sensor accuracy levels, real-time data measurement and capture, and data transfer fluidity from equipment to the visualization and analysis platform. There are two recurrent consultation and acceptance concepts, and the first is interoperability. A complete fleet of underground equipment can now be managed by support teams with the same web platform, regardless of the company, model, or type. The second is bespoke reports by level of information and specific role. The needs and details vary from one role to another, and time is golden, so it needs to be used efficiently and not squandered in generating spreadsheets and manual reports.

- People. The human and cultural factors need attention while carrying out change management work programs and there must be focus on facilitating a successful implementation. It is part of human nature to have some resistance to the unknown, which breaks paradigms and generates new ways of doing things. This is in some cases intensified by intrinsic cultural factors specific to each organization and/or working group. Age is also a factor to consider, because older people have some resistance or lack of familiarity with technology and new ways of doing things.

- Processes. When it comes to implementing technologies these must be included, but indeed they will be a failure. Generating a report to generate a report, without an understanding the reason is not acceptable. A report must have a reason and be understood by everyone involved in the process. For this reason, several communication alternatives were developed within the project in order to be accountable to all stakeholders within the organization. In addition to being defined, responsibilities, periodicities, and backups in cases of absence must continue no matter who is in charge. For KPI management, as previously noted, the system captures a large amount of information. Ideally this is used to define admissible operating parameters and management objectives and to facilitate continuous improvement. There is no possible comparison point if there are no measurement and definition indicators for management.

Table 1 shows a summary of the main key variables to be considered when implementing a telemetry project: 
Table 1 Summary of key points for implementation of telemetry projects

\begin{tabular}{|c|c|c|}
\hline Technology & People & Processes \\
\hline $\begin{array}{c}\text { - Integration with other platforms } \\
\text { (Deswik, PCBC), ERPs, IT systems \& } \\
\text { processes } \\
\text { - Technical feasibility, coverage and } \\
\text { fluidity in the data flow with UG } \\
\text { wireless systems (Wi-Fi network, } \\
\text { 5G, LTE, other) } \\
\text { - Interoperability and multi-brand } \\
\text { concepts } \\
\text { - Real-time and where required } \\
\text { (cloud computing) } \\
\text { - Level of information in reporting } \\
\text { according to role (mine manager, } \\
\text { shift supervisor, service manager) } \\
\text { - Life and durability of sensors } \\
\text { - Information security protocols } \\
\text { - Flexible definition of KPIs and } \\
\text { model control variables } \\
\text { - Benchmarking and implementing } \\
\text { industry best practices } \\
\text { - Feedback on technology } \\
\text { improvements } \\
\text { - Feasibility of new requirements } \\
\text { and developments } \\
\text { - Information feasibility, non- } \\
\text { standardized data }\end{array}$ & $\begin{array}{l}\text { - Management commitment. } \\
\text { Leading the initiative and driving } \\
\text { organizational change } \\
\text { - Identify expectations for the } \\
\text { system } \\
\text { - There must be a project manager } \\
\text { on both parties (owner/supplier) } \\
\text { who generates commitment } \\
\text { - Clearly defined scope and } \\
\text { responsibilities } \\
\text { - Communication and } \\
\text { coordination between } \\
\text { stakeholders } \\
\text { - Barriers to entry: } \\
\text { *Resistance to change } \\
\text { *Control is worse } \rightarrow \text { Sabotage, } \\
\text { negative perception against the } \\
\text { project (e.g. sensor and on-board } \\
\text { instrumentation destruction for } \\
\text { fear of being controlled) } \\
\text { *Ignorance about telemetry as a } \\
\text { technology } \\
\text { *The new is bad, it doesn't work } \\
\text { *Often older people don't } \\
\text { understand technology } \\
\text { - Workshop for new } \\
\text { apprenticeships and enrolees } \\
\text { - Periodic field visits and service } \\
\text { audits to check equipment status, } \\
\text { shift managers, and analysts, and } \\
\text { answer operator doubts } \\
\text { - Regular meetings with different } \\
\text { stakeholders }\end{array}$ & $\begin{array}{c}\text { - Manual vs automated processes. } \\
\text { Avoid administrative tasks and } \\
\text { manipulation of data } \\
\text {-Technology must go hand in hand } \\
\text { with the process } \\
\text { - If there isn't a process one must } \\
\text { be created } \\
\text { - If it exists it must be modified or } \\
\text { adapted } \\
\text {-Managed by KPIs } \\
\text { - Protocol for internal solution } \\
\text { activation } \\
\text { - Global fleet management by } \\
\text { model-serial number } \\
\text { - Identifying problems by cause and } \\
\text { effect } \\
\text { - Clear definition and understanding } \\
\text { of the concept of operation } \\
\text { - Monitoring to check usage of the } \\
\text { platform } \\
\text { - Continuous support with data } \\
\text { center (See section apart) } \\
\text { - Ensuring that the content } \\
\text { of the information displayed } \\
\text { on the platform makes sense } \\
\text { and is understood by all users/ } \\
\text { stakeholders } \\
\text { - Lack of visibility and complexity in } \\
\text { diagnosing equipment failures }\end{array}$ \\
\hline
\end{tabular}

\section{$4 \quad$ Case Studies}

\subsection{Hecla - Casa Berardi, Canada}

\subsubsection{Background}

Hecla Mining's Casa Berardi is a gold mine and mill located in western Québec, some 95 kilometers north of La Serre. In 2018, the mine produced 130,647 ounces of gold and it has been in operation since 1989. It currently employs about 580 people.

\subsubsection{The challenge}

Casa Berardi wanted to improve their productivity by reducing maintenance costs. To do that, the mine needed to cut down wear and tear on their equipment and they also needed to be able to monitor machines to catch equipment problems before they became major failures.

Brake tests are an important step in keeping operators safe. Performing them daily ensures that the machine's brakes are in proper operating condition. Additionally, preventing transmission overspeeds is 
critical to preventing serious damage to the transmission. With sensors, Hecla was able to monitor these in real time, so they were able to see when tests were being performed correctly.

Newtrax (2019) was also able to install a sensor suite that gave mechanical technicians the ability to more easily identify problems when a machine is brought in. It also makes it easier to monitor equipment to avoid problems before they develop into something more serious.

\subsubsection{Testimonies}

- "In 2017, we started the telemetry project. We have installed a solution on 24 vehicles, on trucks, on LHD, in order to collect data remotely in our vehicles in operation" — Jérémie Frenette - Senior Electrical Supervisor - Project Manager - Hecla Québec - Casa Berardi Mine.

- "A vehicle, in the past, when you had to, for example, find an intermittent issue, we were stuck with it for up to two weeks to recreate the problem, to diagnose it" - Vincent Corriveau - Mobile Equipment Mechanic - Hecla Québec - Casa Berardi Mine.

- "When we started, we put a lot of emphasis on the behavior of our operators and one of the types of data we were looking for was transmission abuse. And we started to see that some operators didn't know why they were causing these alarms. So they were trained and changed their ways of operating the machines and that brought great results." - Sonny Melancon Control Room Operator - Hecla Québec - Casa Berardi Mine.

- "Now we can connect directly to the computer of the machine and can diagnose it when it is a lot farther in the mine site because the data of the machine is available in real time to compare with the other machines." - Vincent Corriveau - Mobile Equipment Mechanic - Hecla Québec Casa Berardi Mine

- "If we compare with last year, where we were not able to go above our budgeted quantities for our underground mine, this year we are $4 \%$ above our targets. And of this $4 \%$, I would say that half of that is attributed to telemetry. If we compare an equivalent period of nine months in 2017 to 2018 , we noticed that, for the LHDs especially, an increase in availability of $8 \% . "$

\subsubsection{The outcome}

- Savings of 30 minutes per shift thanks to fewer delays.

- Transmission overspeeds dropped by $10 \%$ with a 50\% decrease in transmission abuse.

- Savings of $7.8 \%$ in total in the maintenance costs per each tonne transported.

- The mine was able to process about $2 \%$ over its budgeted quantities for the year.

- LHD availability increased by $8 \%$.

\subsection{Glencore Matagami mine}

\subsubsection{Background}

Located in the heart of the boreal forest in the Nord-du-Québec region, Glencore Matagami Mine is located about $10 \mathrm{~km}$ west of the city of Matagami.

The Bracemac and McLeod deposits consist of several lenses rich in zinc and copper that also have low silver and gold grades.

Glencore Matagami Mine first implemented the Newtrax Mobile Equipment Telemetry system back in 2016 on their trucks and LHDs in order to maximize their haulage efficiency. 


\subsubsection{The challenge}

Glencore Matagami mine's biggest challenge is the long haulage distance they have to travel every day to transport the ore out of the mine. They currently have to travel $8 \mathrm{~km}$ (4.9 miles) by truck, which is a significant distance, and this will be increased to $10.4 \mathrm{~km}$ (6.5 miles) in the coming years.

Long distance haulage means that each cycle makes a huge difference in productivity. Maximizing the load on each truck is essential for this mine.

\subsubsection{Common roadblocks}

Most underground mines interested in digitizing their mines don't start from a clean slate, and often grow with acquisitions. However, they frequently encounter stumbling blocks on their road to success which include:

- Non-standardized data. Multiple equipment manufacturers for mobile equipment, coupled with data that isn't standardized makes it tough to pull everything together.

- Lack of data at the face. It's hard to get an accurate picture of what's going on in the mine if you don't have live data from the mine's faces-including those under development.

- Incomplete analysis. Maybe you do have all the data you need, you're just unsure what to do with it. Analytical systems can help you take all that data you have and turn it into actionable insights.

The Newtrax mobile equipment telemetry system, which is compatible with all equipment brands and models, was easily integrated to Matagami mine's mixed fleet of trucks and LHDs. Glencore Matagami used the system in multiple ways:

- To monitor the standard production times of equipment.

- To calculate utilization of ore haulage.

- To calculate the Overall Equipment Effectiveness (OEE).

- To calculate loads per cycle.

\subsubsection{The outcome}

- $6 \%$ increase in utilization on their ore haulage.

- $4 \%$ increase on the overall equipment effectiveness.

- $5 \%$ increase on loads per cycle.

\subsubsection{Operational outcome}

The standard production time report, which shows operators the activity of each piece of mobile equipment in a period of 24 hours, has enabled the underground operations personnel to identify problem areas quickly, discuss them as a group before the start of shift, and apply identified improvements in order to increase their OEE down the line.

\subsubsection{Management outcome}

From a managerial perspective, the Newtrax system has enabled the team to make decisions based on facts that are measurable rather than off of perceptions and gut feelings. Since implementing this, they have been able to raise their average tonnage from 55 tonnes to 60 tonnes which has increased productivity; especially given the long haulage distances they are facing. 


\section{$5 \quad$ Benefits}

Using technologies such as telemetry, it is possible to keep track of fleets and underground mining operations, regardless of distance, to optimize processes. This system places sensors on vehicles and assets to monitor their performance in real-time through a system of reports, notifications, alarms and associated events. Some benefits identified in customer deployment experiences are summarized below.

- Allows remote contact and monitoring from any device with internet connection for all units in the fleet, as well as mining assets and equipment. This benefits mine safety and production.

- Enables a preventive maintenance vs corrective maintenance approach.

- Allows direct input to set up maintenance plans.

- Reduces component wear, and equipment maintenance and operating costs.

- Optimizes diagnosis time for problems and equipment failures.

- Promotes communication within the organization.

- Facilitates transparency and control of information by concentrating on a single source.

- Provides real-time information on any device with internet access for decision-making when action needs to be taken.

- Creates a scalable solution from one pilot test to a total multi-brand fleet.

- Ensures visibility and control of the process.

- Allows verification and improvement of equipment productivity.

- Extends the life of equipment and components.

- Reduces exposure to risks and increases safety and ergonomics for people.

- Promotes efficient use of resources and a sustainable ecosystem.

- Reduces administrative tasks and manual data capture from operations and equipment.

- Promotes continuous operation and increases availability and use of equipment.

- Enables improvement of equipment operation with operator event notifications.

- Provides alarm reports and makes visible anomalous operating component conditions.

- Analyzes cause and effect based on disaggregated and historical behavioral information.

- Facilitates the identification of potential faults and anomalies with qualitative expert judgement thereby avoiding major component damage.

- Records downtime and refueling practices and analyzes fuel use for each piece of equipment.

- Facilitates result assessments and decision-making through management indicators based on data collected.

- Checks operator performance and generates training programs by areas of improvement.

- Scales from a connected fleet (pilot) to a full fleet of underground equipment.

\section{Conclusions}

The path to digital transformation, especially for technologies like telemetry, is one that mining companies have been increasingly taking. Due to the benefits in terms of productivity, cost efficiency and safety, surely many more will join over time. The Hecla Casa Berardi Case Study demonstrated a reduction in maintenance cost of $7.8 \%$, improved the loader productivity by $8 \%$, and improved safety with a drop of $10 \%$ in overspeeds. However, one of the risks of this implementation is the uncertainty of how effective the digital transformation will be. There is no one-of-a-kind path to success. There are various models for project implementation, and all of them consider, to some degree, the three approaches to all change 
management: technology, processes and people. In addition, the SIC-based GMG best practice guide for mining companies provides strong reference for the way forward. On the digital path it is necessary to adapt agilely with a continual improvement approach in order to ensure the project success.

\section{Acknowledgements}

Thanks to all people who added a degree of experience to the creation of this paper, and for contributing their point of view. Special thanks to Hector Medina, Boris Burgos and Lior Herman.

\section{References}

Global Mining Guidelines Group 2019, 'Guidelines for Implementing Short Interval Control in Underground Mining Operations', Retrieved from Global Mining Guidelines Group: https://gmggroup.org/publication-guideline-implementing-shortinterval-control-underground-mining-operations/ [6 June 2019]

Mckinsey 2015, 'How digital innovation can improve mining productivity', https://www.mckinsey.com/industries/metals-andmining/our-insights/how-digital-innovation-can-improve-mining-productivity\#, [November 2015]

Newtrax 2019, 'Case studies', https://www.newtrax.com/case-studies/

Sandvik, 'My Sandvik productivity' https://www.rocktechnology.sandvik/en/parts-and-services/my-sandvik-digital-servicesolutions/my-sandvik-productivity/,https://www.youtube.com/watch?v=nrZdGWGd3cl\&app=desktop, https:// solidground.sandvik/solutions-digital-future/

World Economic Forum in collaboration with Accenture 2017, 'Mining and Metals: digital transformation and the industry's 'new normal', https://reports.weforum.org/digital-transformation/mining-and-metals-digital-transformation-and-theindustrys-new-normal/, [January 2017] 